\title{
AMBIENTE FRIO E O ACONCHEGO DA EQUIPE DE UMA UTI INFANTIL: compartilhando experiências do estágio supervisionado de enfeermagem na UTI infantil
}

\author{
Rosângela Vidal de NEGREIROS ${ }^{1}$ \\ Thaynara Souto Vasconcelos LINS ${ }^{2}$ \\ Rosenilda Dias da SILVA ${ }^{3}$ \\ Raine Danyelle Vieira de SOUSA ${ }^{4}$ \\ Larissa Ferreira Araújo PAZ ${ }^{5}$ \\ Ruan Tcharle Pereira de SOUZA ${ }^{6}$
}

\footnotetext{
${ }^{1}$ Mestre em Enfermagem, docente do curso de Enfermagem, da Universidade federal de Campina Grande (UFCG), do CCBS. E-mail: rosangelavn@ufcg.edu.br.

${ }^{2}$ Discente do curso de graduação em enfermagem do CCBS da UFCG. E-mail: thaysv_10@yahoo.com.br

${ }^{3}$ Graduada do curso de Enfermagem na Universidade Federal de Campina Grande (UFCG), do CCBS.

E-mail: rosenilda@797gmail.com.

${ }^{4}$ Graduada do curso de Enfermagem na Universidade Federal de Campina Grande (UFCG), do CCBS.

E-mail: raine_sousa@hotmail.com

${ }^{5}$ Graduada do curso de Enfermagem na Universidade Federal de Campina Grande (UFCG), do CCBS.

E-mail: larissaaraujopaz@gmail.com

${ }^{6}$ Discente do curso de graduação em enfermagem do CCBS da UFCG. E-mail: ruantcharles@ gmail.com
}

Recebido em: 05/05/2016 - Aprovado em: 22/11/2017 - Disponibilizado em: 30/12/2017

\begin{abstract}
RESUMO
A experiência no estágio supervisionado proporciona ao acadêmico de enfermagem o desenvolvimento de capacidades teórico-práticas, habilidades e competências importantes à sua formação enquanto enfermeiro da assistência hospitalar. Tendo como objetivo compartilhar experiências vividas na Unidade de Terapia Intensiva (UTI) infantil durante o estagio no Hospital Universitário Alcides Carneiro, trata-se de um relato de experiência vivenciado no curso de Enfermagem da Universidade Federal de Campina Grande, com supervisão direta do enfermeiro do setor e indireta do coordenador de estagio. O período de estágio na UTI infantil proporcionou enriquecida fase de aprendizagem e crescimento pessoal, oferecendo a oportunidade de presenciar a importância da teoria adquirida durante o curso, ao desenvolver as práticas no serviço, assim como a atualização contínua nos estudos de modo aperfeiçoar as técnicas do cuidado intensivo na UTI. Destacando-se os profissionais que atuam no setor infantil com seu extremo comprometimento com o paciente, desenvolvendo um trabalho de acolhimento, atendimento ao usuário infantil em estado grave e familiares, com apoio psicossocial e terapêutico em um ambiente que só lida com o sofrimento humano, em momentos em que o paciente está em situação de gravidade, de comprometimento da vida. Tornando-se uma experiência única e gratificante, evidenciando um maior enriquecimento profissional, contribuindo para a humanização do atendimento na enfermagem, autoconfiança e segurança no desenvolver do atendimento ao paciente que necessita de cuidados, o que contribuirá para a assistência de enfermagem com qualidade e comprometimento profissional.
\end{abstract}

Palavras-chave: Estágio supervisionado. Enfermagem. Unidade de Terapia Intensiva. Cuidado. Saúde.

\footnotetext{
ABSTRACT

Experience in supervised interniship provides the nursing academic the development of theoretical and practical abilities, important skills and competencies to their formation as nurses in hospital care. Aiming to share experiences lived in the Pediatric Intensive Care Unit (PICU) during the internship at University Hospital Alcides Carneiro, it is an experience report witnessed at Nursing school of Federal University of Campina Grande, under the direct supervision of 
the nurse section and indirectly of the internship coordinator. The interniship period in PICU provided enriched phase of learning and personal growth, offering the opportunity to witness the importance of theory acquired during the course, by developing practices in service, as well as continuous updating in studies in order to improve the techniques of intensive care in the PICU. Standing out professionals that work in the children's sector with its extreme commitment to the patient, developing a host work, care to pediatric patients in critical condition and relatives, with psychosocial and therapeutic support in an environment that only deals with human suffering, at times when the patient is in serious condition, impairment in life. Becoming a unique and rewarding experience, demonstrating a greater professional development, contributing to the humanization of care in nursing, self-confidence and security at the development of the patient attendance that needs care, which shall contribute to nursing assistance with quality and professional commitment.

Keywords: Supervised interniship. Nursing. Pediatric Intensive Care Unit. Care. Health.

\section{INTRODUÇÃO}

O estágio supervisionado é um componente curricular obrigatório na graduação em enfermagem, oferecido no último semestre do curso e com carga horaria de 420 horas, destinado à elaboração do cronograma, supervisão do estagiário e planejamento das atividades, reuniões tutoriais, estudos comparados, reuniões de aperfeiçoamento com a expressiva participação dos graduandos em enfermagem, dos profissionais do serviço de saúde, de um coordenador de estagio e todos os envolvidos (RODRIGUES; TAVARES, 2012).

$$
\text { Para realização do estagio foi }
$$
selecionado os setores do Hospital Universitário Alcides Carneiro (HUAC), onde os alunos são distribuídos, sendo a UTI infantil local destinado ao estagio de seis horas diárias, de segunda a sexta, com supervisão direta do enfermeiro assistencial e indireta do coordenador de estágio, onde o aluno presta assistência aos usuários, desenvolve ações, gerenciar, coordena a equipe de enfermagem, dentre outras atividades privativas do enfermeiro.
De acordo com a Lei $\mathrm{n}^{\circ} 11.788$, de 25 de Setembro de 2008 (BRASIL, 2008) estágio é um ato supervisionado que se desenvolve no ambiente de trabalho intencionando a preparação para o trabalho produtivo.

O HUAC fica localizado no município de Campina Grande - Paraíba, oferecendo à comunidade serviços de média e alta complexidade na atenção a saúde, sendo referencia em doenças infecto contagiosas e anemia falciforme, com atendimento em varias clínicas adultas e infantil.

De acordo com a portaria 1.071 de 04 de junho de 2005 a UTI é um local de grande especialização e tecnologia, constituindo-se um espaço laboral destinado aos profissionais da saúde, principalmente médicos e enfermeiros, que possuem alto nível de conhecimento, habilidades e destreza diferenciados para a realização de procedimentos que definem o limite entre a vida e a morte das pessoas (BRASIL, 2005).

$\mathrm{Na}$ UTI pode-se acompanhar a humanização na assistência, representando assim, uma oportunidade de acompanhar o acolhimento e a valorização do usuário, na 
produção de cuidados capaz de conciliar tecnologia disponível com a promoção de respeito ético, tornando espaços favoráveis ao exercício técnico e à satisfação dos profissionais e usuários, incremento de educação continuada dos servidores, sobrecarga de trabalho, assistência mecanizada e tecnicista, desconsiderando, os princípios da humanização do cuidado. (LEITE; SILVA; PADILHA, 2012).

A UTI divide-se de acordo com a modalidade e pela faixa etária em: Neonatal destinado ao atendimento de pacientes com idade de 0 a 28 dias; pediátrico atendimento a idade de 29 dias a 18 anos incompletos, UTI adulto destinado a pacientes com idade acima de 14 anos, enquanto que pacientes entre 14 a 18 anos incompletos podem ser atendidos nos Serviços de Tratamento Intensivo Adulto ou Pediátrico (BRASIL, 2010).

De acordo com a portaria $\mathrm{n}^{\circ} 930$ de 10 de maio de 2012 a unidade neonatal é um serviço de internação responsável pelo cuidado integral ao recém-nascido grave ou potencialmente grave, dotado de estruturas assistenciais que possuam condições técnicas adequadas à prestação de assistência especializada, incluindo instalações físicas, equipamentos e recursos humanos (BRASIL, 2012).

As modernas unidades de terapia intensiva neonatal (UTIN), equipadas com tecnologia de ponta são um marco na assistência ao recém-nascido de risco, contribuindo para sua sobrevida, tendo como foco a assistência aos aspectos biológicos. (CHAGAS et. al, 2008).

A UTI Pediátrica Mista, de acordo com a portaria $\mathrm{n}^{\mathrm{o}} 7$ de 2010 aponta os requisitos que devem atender a procedimentos intensivos, recursos humanos, assistenciais e materiais estabelecidos para UTI pediátrica e neonatal concomitantemente (BRASIL, 2010).

Nesse contexto a UTI infantil do HUAC se classifica como mista, sendo neonatal e infantil. Atende diversas patologias, que demandam cuidados diretos a pacientes graves e com risco de vida. Trata-se de um setor dinâmico, com vários protocolos de atendimento de urgência, emergência e avançados, normas e rotinas, checagem de medicamentos, exames laboratoriais, dentre outros.

Os usuários fazem uso de vários procedimentos invasivos como, tubo orotraqueal, traqueostomia, gastrotomia, acesso venoso central, drenos, ventilação mecânica invasiva, monitorização contínua, equipamentos especiais como baby hood, entre outros, sendo importante o seu acompanhamento para a aprendizagem dos alunos, conferindo a oportunidade de realizarem vários procedimentos invasivos na atenção ao paciente critico.

Assim, diante do exposto, o objetivo desse estudo é compartilhar as experiências vividas no estágio supervisionado na UTI 
infantil, devido ao amplo campo para de atividades práticas, vivenciando a dinâmica multidimensional no atendimento ao paciente grave.

\section{METODOLOGIA}

Trata-se de um relato de experiência, vivenciado por discentes do curso de bacharelado em Enfermagem da UFCG, campus CCBS, durante o estágio supervisionado, desenvolvido no HUAC, disciplina da grade curricular do $10^{\circ}$ período do curso, carga horária obrigatória de 420 horas, com 60 turnos de seis horas diárias, mais reuniões tutoriais, realizado entre abril a junho de 2015, com supervisão direta de enfermeiros do setor.

A UTI é dividida em neonatal e infantil, na UTI infantil funcionam todos os leitos, recebendo crianças acima de 29 dias até a adolescência. A neonatal recebe crianças de até 29 dias de nascimento como preconiza o Ministério da Saúde.

A UTI atende vários municípios da região paraibana, recebendo crianças que necessitam de cuidados intensivos. Quanto aos recursos humanos temos enfermeiro coordenador, enfermeiro assistencial, técnicos de enfermagem em plantões de 12. Além de médico intensivista pediátrico, equipe de fisioterapia e alunos da UFCG que são acompanhados pela equipe plantonista.

O enfermeiro é responsável pela gestão e gerenciamento de matérias da farmácia, central de material esterilizado (CME), serviço de gráfica, material de limpeza, inspeção, funcionamento e montagem de respiradores, aparelhos de oxigenoterapia, monitorização, equipamentos eletrônicos, coordena e supervisiona a equipe de enfermagem e oferecer assistência aos familiares das crianças.

\section{RESULTADOS E DISCUSSÃO}

$\mathrm{Na}$ UTI infantil, os profissionais e estagiários continuamente encontram-se em assistência de enfermagem os pacientes graves e com risco de vida, serviços burocráticos, tornando um cenário promissor e amplo de conhecimento para estagiários de enfermagem no que concerne a experiência prática.

Quanto a estrutura física temos um ambiente amplo com quatro leitos, leito de isolamento, quatro berços aquecidos, posto de enfermagem, sala de prescrição, balcão, corredor, maca de transporte, banheiro para funcionários, repouso de enfermagem, copa, almoxarifado, expurgo e arsenal.

Com relação aos recursos humanos, em cada plantão temos dois enfermeiros, um técnico diarista, quatro técnicos plantonista em regime de 12 horas, auxiliar de serviços gerais, médico pediatra e fisioterapeuta.

No que concerne aos recursos materiais, no posto de enfermagem contém materiais necessários para realização de 
procedimentos rotineiros, armários para armazenamento de roupas, lençóis, medicamentos, pia para lavagem de mãos, gavetas plásticas, geladeira, carro de parada, armários para materiais de procedimentos, balcão com pastas de impressos.

Os leitos são modernos, camas automáticas, berços aquecidos com fototerapia, respiradores e monitores, poltronas para acompanhantes, aparelho de Raio X portátil, eletrocardiógrafo e mesas auxiliares para procedimentos.

Durante o estágio foram desenvolvidas atividades a clientes graves, com risco de vida, como organização administrativa, inspeção e monitoração de medicamentos, sistematização da assistência de enfermagem, mudança decúbito, assistência à monitoração contínua, aspiração no tubo oro-traquel, cuidados em parada cardiorrespiratória, ventilação mecânica, funcionamento da bomba de infusão continua, administração de medicamentos, coleta de sangue para exames laboratoriais, coleta de Swab nasofaringe, gasometria arterial, cuidados com o corpo pós-morte, gerenciamento da equipe de enfermagem, requisição e suprimento de materiais de rotina, manutenção de leitos e berços aquecidos, montagem de equipamentos de respiradores e monitores cardíacos, teste de glicemia capilar, aplicação de medicamentos, banhos no leito, cuidados ao recém-nascido, exame físico cefalopodálico, sinais vitais, evolução de enfermagem, admissão, oxigenoterapia, organização do posto de enfermagem, do arsenal, preparo e administração de medicamentos, curativos diversos, cuidados com dreno de sucção, aparelhos de fototerapia, Baby Hood, eletrocardiograma, antibióticos em uso, em refrigeração, controle e inspeção dos medicamentos psicotrópicos e cuidados com acesso venoso central.

Realização de técnicas para esterilização de materiais, habilidades na dinâmica de grupos, controle de antibióticos, imunobiológicos, acompanhamento de pacientes para exames de Raios-X, exames laboratoriais, eletrocardiograma, relato de ocorrência do plantão e passagem de plantão, ações de conforto físico e psicológico de familiares dos internos na UTI infantil, esclarecendo duvidas quanto a providencias no pós-morte, medidas de conforto, autocuidado, mudança de decúbito para prevenção de úlceras por pressão, medidas de prevenção de quedas, apoio emocional aos familiares.

As atividades gerenciais e assistenciais foram importantes, pois durante o decorrer do curso de enfermagem não tínhamos vivenciado uma assistência tão completa, abordando a humanização no cuidado em enfermagem, capacidade operacional no serviço de saúde, iniciativa, ensinamentos esses, que proporcionam ao discente um compromisso com todos aqueles que precisam de UTI. 
O estagio na UTI infantil proporcionou grande aprendizagem e crescimento profissional, por desenvolver um amplo conhecimento na área, com a oportunidade de ser supervisionada por excelentes profissionais, com ampla prática profissional, aliado ao compromisso, da coordenadora do setor e dos outros enfermeiros, que são extremamente preparados para desenvolver suas atribuições na UTI infantil, à maioria tem residência, especialização ou fazem mestrado na área, que despertou o interesse em estudar sobre a área, investir em materiais didáticos, minicursos, capacitações sobre atendimento a pacientes graves e com risco de vida.

Os encontros tutoriais, realizados nas sextas feiras, contribuíram como oportunidade para retirar dúvidas, capacitando mais os graduandos de enfermagem. A coordenação sempre proporcionou apoio fundamental, questionando a equipe do setor sobre $\mathrm{o}$ desempenho dos estagiários, proporcionando interesse dos alunos no desenvolvimento de suas atividades.

Como ponto posito do estagio, a receptividade dos profissionais do HUAC se destaca, quanto ao desempenho e capacidade da coordenação, em articula entre enfermeiros assistenciais, profissionais da equipe, usuários dos serviços e alunos, tornando um local excelente, pela cooperação, integração, capacitação técnica, prestando um serviço extremamente importante na transmissão de conhecimento, e facilitando o desempenho dos discentes. Tornando-se fundamental o relacionamento interpessoal positivo, harmonioso, competente e com respeito no desempenho da profissão.

Assim, a imersão no mercado de trabalho torna-se gratificante, impõe desafios quanto à superação de barreiras para o desempenho de atividades profissionais com excelência dos futuros enfermeiros.

\section{Análise crítica da vivência}

O estágio foi relevante, extremamente oportuno, pois proporcionou ao discente a capacidade de desenvolver habilidades de contato com os usuários no serviço hospitalar, enquanto membro coordenador da equipe de enfermagem, garantindo maior desempenho nas relações interpessoais, desenvolvendo a capacidade prática, com foco na assistência hospitalar de média e alta complexidade, aperfeiçoando o discente a desenvolver autoavaliação enquanto gerente de um serviço, garantindo a saúde dos pacientes, proporcionando ao aluno o desempenho de atividades práticas extensa, tendo em vista a vivencia continua no serviço hospitalar.

A disciplina é considerada positiva e gratificante, pois permiti ao discente formar suas vivencias práticas enquanto membro de uma profissão que exige teoria, prática e nível psicológico aguçado, garantindo um 
profissional competente e comprometido com a profissão.

Como ponto negativo no estagio, considero a falta da implementação eficiente da Sistematização da Assistência de Enfermagem (SAE) pelos profissionais, por ser privativo e de sua competência, contribuindo para organizar o trabalho do enfermeiro.

De acordo com a resolução 358/2009, a SAE e o processo de enfermagem representam uma assistência de modo planejado, com desenvolvimento de competências privativas da profissão e o compromisso de uma assistência de enfermagem dinâmica, cientifica, obedecendo às etapas de histórico de enfermagem ou coleta de dados, diagnostico, planejamento, implementação e avaliação (COFEN, 2009; CAVALCANTI et al, 2011).

A aplicação da Sistematização da Assistência de Enfermagem, um ato privativo do enfermeiro envolve mais do que uma sequência de passos a ser proposto pelos mesmos, urge dos supracitados profissionais maior familiaridade dos diagnósticos de enfermagem e sensibilidade para adequar as necessidades do cliente às condições de trabalho (SILVA et al, 2011). Sendo importante a associação do conteúdo aprendido em sala de aula na universidade e a prática desenvolvida no estagio supervisionado no HUAC.

\section{CONSIDERAÇÕES FINAIS}

$O$ estagio na UTI infantil foi um desafio enquanto acadêmica de enfermagem proporcionou aumento nos conhecimentos na área, incentivou estudar a temática, assim como realizar atividades práticas de forma intensiva.

O estagio oportunizou uma gama de conhecimentos sobre diversas patologias envolvendo a vida desde o nascimento até a pós-morte no período inicial da vida e como a enfermagem lida com esse acontecimento.

Vale salientar que a confiança depositada no aluno pelos profissionais, dos usuários e familiares do serviço proporcionou investir em maior empenho no desenvolver das atividades na UTI infantil.

No que concerne à organização e coordenação do estagio supervisionado houve um ótimo planejamento das atividades dos docentes, uma preocupação em aumentar o relacionamento no campo de estagio, preocupação com a qualidade da assistência prestada por parte dos alunos, com investimento em aulas teórico-práticas em diversos assuntos pertinentes ao estágio, houve também uma excelente comunicação por parte da coordenação do estagio.

Com relação a UTI infantil é interessante sugerir a efetivação da assistência de enfermagem, criação de protocolos sistematizados pela enfermagem, sendo perceptível enquanto acadêmico de 
enfermagem estudar mais, participar mais de cursos na área de UTI infantil para assim garantir enquanto profissional prestes a sair da Universidade, uma enfermagem potencialmente comprometida com vida daqueles que precisam de atendimento em situações graves e com risco de vida.

Dessa forma evidencia-se que o estagio supervisionado foi extremamente válido em minha vida enquanto profissional de enfermagem, para a formação de um futuro profissional inserido no competitivo mercado de trabalho.

\section{REFERÊNCIAS}

BRASIL. Portaria n ${ }^{\circ}$ 1.071/MS/GM, de 04 de julho de 2005. Determina que a Secretaria de Atenção à Saúde submeta à Consulta Pública a minuta da Política Nacional de Atenção ao Paciente Crítico. Diário Oficial [da] República Federativa do Brasil, Brasília, DF, 04 jul 2005. Disponível em: < http://bvsms.saude.gov.br/bvs/saudelegis/gm/ 2005/prt1071_04_07_2005.html > Acesso em: 15 jun 2015.

. Portaria ${ }^{\circ}$ 930/MS/GM, de 10 de maio de 2012. Define as diretrizes e objetivos para a organização da atenção integral e humanizada ao recém-nascido grave ou potencialmente grave e os critérios de classificação e habilitação de leitos de Unidade Neonatal noâmbito do Sistema Único de Saúde (SUS). Diário Oficial [da] República Federativa do Brasil, Brasília, DF, 10 mai 2012. Disponível em:

<http://bvsms.saude.gov.br/bvs/saudelegis/gm /2012/prt0930_10_05_2012.html>Acesso em: 18 jun 2015.

\section{BRASIL. RESOLUÇÃO ANVISANº7/2010.}

Dispõe sobre os requisitos mínimos para funcionamentos de unidades de terapia intensiva e dá outras providencias. Disponível em: http://bvsms.saude.gov.br/bvs/saudelegis/anvi sa/2010/res0007_24_02_2010.html. Acesso em 02 de jun.2015.

CAVALCANTI, R. B. et. al. Experiências de Sistematização da Assistência de Enfermagem no Brasil: um estudo bibliográfico. Enferm. UFSM, p.461-471, Rio Grande do Sul, 2011. Disponível em: http://cascavel.ufsm.br/revistas/ojs2.2.2/index.php/reufsm/article/viewFile/2832/ 2396. Acesso em: 30 de mai.2015.

CHAGAS, R.I. A et al. Análise dos dos fatores obstétricos, socioeconômico e comportamentais que determinam a frequência de recém-nascidos pré-termo em UTI neonatal. Revista da Sociedade Brasileira de Enfermeiros Pediatras, v.9, n.1. Pernambuco, 2008. Disponível em: http://www.sobep.org.br/revista/component/zi ne/article/112-anlise-dos-fatores-obsttricossocioeconmicos-e-comportamentais-quedeterminam-a-frequncia-de-recm-nascidos-prtermos-em-uti-neonatal.html. Acesso em: 02 de jun.2015.

\section{COFEN. RESOLUÇÃO COFEN N ${ }^{\circ}$}

358/2009. Dispõe sobre a Sistematização da

Assistência de Enfermagem e a

Implementação do Processo de Enfermagem em ambientes públicos e Privados, e dá outras providências. Disponível em: <http:// http://www.cofen.gov.br/resoluo-cofen3582009_4384.html. Acesso em 02 de jun.2015.

RODRIGUES, L.M. S; TAVARES, C.M.M. Estágio Supervisionado de Enfermagem na atenção básica: $\mathrm{O}$ planejamento dialógico como dispositivo do processo ensinoaprendizagem, Rev. Rene, p.1075-1083, Rio de Janeiro, 2012. Disponível em: http://pesquisa.bvs.br/brasil/resource/pt/lil679867. Acesso em: 01 de jun.2015.

LEITE, I.R. L; SILVA, G.R.F.; PADILHA, K.G. Nursing Activities Score e demanda de trabalho de enfermagem em terapia intensiva. Acta paul. enferm. v.25, n.6, São Paulo, 2012. Disponível em: 
http://www.scielo.br/scielo.php?script=sci_art text\&pid=S0103-21002012000600003.

Acesso em 26 de mai.2015.

SILVA, E.G. C et al. O conhecimento do enfermeiro sobre a Sistematização da Assistência de Enfermagem: da teoria à prática. Rev. Esc. Enferm USP, p.13801386, São Paulo, 2011. Disponível em: http://www.scielo.br/pdf/reeusp/v45n6/v45n6 a15.pdf. Acesso em: 02 de jun.2015. 